\title{
OPERAÇÃO DO MAIOR CIRCUITO DE REMOAGEM DO MUNDO UTILIZANDO VERTIMILL*
}

\author{
Douglas Batista Mazzinghy ${ }^{1}$ \\ José Francisco Cabello Russo ${ }^{1}$ \\ Paula Moreira Mol Pereira ${ }^{2}$ \\ Luís Cláuzio de Rennó Machado² \\ Valdiney Chaves ${ }^{2}$ \\ Nemer Saib Neto ${ }^{2}$ \\ Luís Marcelo Marques Tavares ${ }^{3}$
}

\section{Resumo}

O Projeto Minas-Rio possui o maior circuito de remoagem do mundo com o uso de moinho Vertimill. O circuito foi comissionado no final de 2014 e vem apresentando desempenho conforme projetado. São duas linhas de remoagem sendo que cada linha possui oito moinhos Vertimill modelo Metso VTM-1500 e quatro baterias de ciclones, totalizando dezesseis moinhos e oito baterias de ciclones. $O$ circuito é do tipo reverso e são utilizadas bolas de alto cromo de $12,5 \mathrm{~mm}$ de diâmetro como carga moedora. O moinho Vertimill é bem robusto e não apresentou problemas maiores durante os primeiros seis meses de operação. O presente trabalho faz uma avalição da operação destes equipamentos e compara os dados de projeto com os resultados industriais obtidos através de campanhas de amostragens.

Palavras-chave: Vertimill; Remoagem; Minério de ferro; Itabirito; Minas-Rio.

\begin{abstract} AROUND THE WORLD UTILISING VERTIMILL

The Minas-Rio Project has the largest regrind circuit around the world using Vertimill. The circuit was commissioned in the end of 2014 and has shown performance as designed. It consists of two regrind lines with each line having eight Vertimill VTM1500 model supplied by Metso and four cyclones clusters, totaling sixteen mills and eight cyclones clusters. The circuit is configured in reverse mode and has been loaded with $12.5 \mathrm{~mm}$ high chrome balls. The Vertimill's are very robust and presented no major problems during the first six months of operation. This paper assesses the operation of these mills and compares the design data with industrial data collected during sampling campaigns.
\end{abstract}

OPERATION OF THE LARGEST REGRIND CIRCUIT

Keywords: Vertimill; Regrinding; Iron ore; Itabirite; Minas-Rio.

1 Desenvolvimento de Processos, Anglo American Minério de Ferro Brasil, Conceição do Mato Dentro, MG, Brasil.

2 Operação, Anglo American Minério de Ferro Brasil, Conceição do Mato Dentro, MG, Brasil.

3 Professor Titular, Laboratório de Tecnologia Mineral, Programa de Engenharia Metalúrgica e de Materiais, COPPE, Universidade Federal do Rio de Janeiro, RJ, Brasil. 


\section{INTRODUÇÃO}

\subsection{Minas-Rio}

O Projeto Minas-Rio, de propriedade da Anglo American, iniciou as suas operações no final de 2014. A planta de processamento mineral possui etapas de britagem primária e secundária, prensas de rolos e circuito de moagem com moinhos de bolas. A deslamagem do material é realizada por meio de hidrociclones e a concentração através de flotação reversa em tanques. As etapas de separação sólido-líquido são realizadas em espessadores e a remoagem do Pellet Feed é realizada usando moinhos Vertimill. O Pellet Feed é bombeado através do maior mineroduto do mundo com mais de $525 \mathrm{~km}$ de extensão. Já no porto o material é filtrado usando filtros cerâmicos, estocado e embarcado para o mercado externo. A Figura 1 apresenta o fluxograma da planta de processamento mineral do Minas-Rio.

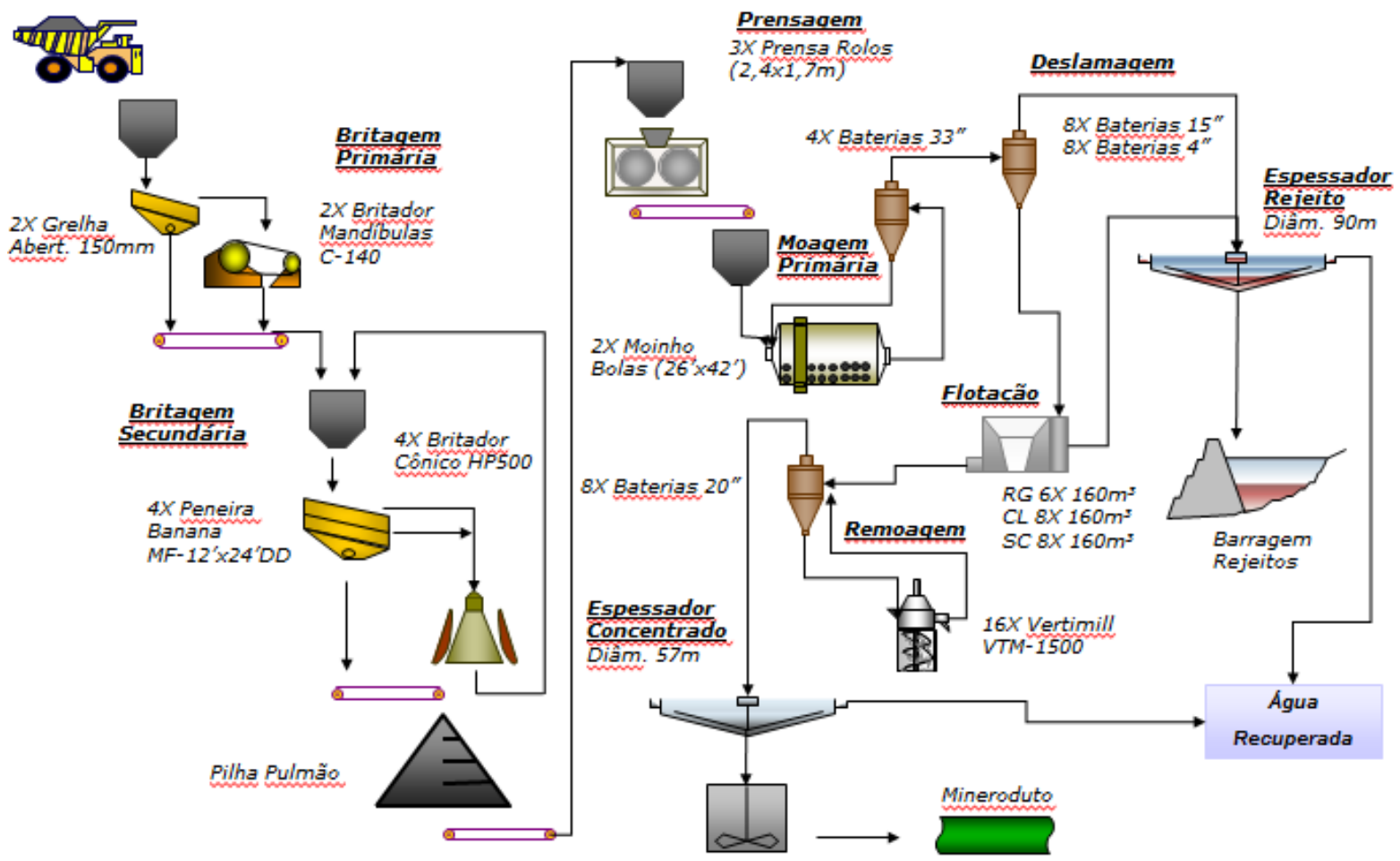

Figura 1. Fluxograma da planta de processamento mineral do Minas-Rio.

O Minas-Rio encontra-se na fase de ramp up e vem até o momento operando conforme planejado.

\subsection{Vertimill}

O moinho Vertimill é constituído de um cilindro orientado na vertical carregado com corpos moedores. Uma espiral instalada no centro deste cilindro é responsável pela movimentação dos corpos moedores que promovem a redução das partículas através do impacto e atrito entre elas. O fluxo de polpa pode ser alimentado pelo topo do moinho ou pela parte inferior do mesmo. O produto do moinho é transbordado pelo topo. A zona entre o leito de bolas e o topo do moinho por onde o produto é transbordado forma a zona de classificação interna do Vertimill [1]. 
Os moinhos verticais se diferenciam dos moinhos horizontais devido à frequência e intensidade dos impactos entre os corpos moedores. Através de modelamento usando o método dos elementos discretos (DEM - Discrete Element Method) é possível prever o comportamento de cada corpo moedor dentro dos moinhos. Basicamente, o moinho vertical apresenta maior número de colisões entre os corpos moedores, porém com menor intensidade enquanto os moinhos tubulares rotativos apresentaram um comportamento oposto, com menor número de colisões entre os corpos moedores, porém de maior intensidade [2].

O moinho Vertimill já vem sendo utilizado na etapa de remoagem em algumas operações no Brasil: Sossego e Salobo da Vale S.A, Germano da Samarco, Paracatu da Kinross, Maracá da Yamana, Jaguarari da Caraíba e, agora, Minas-Rio da Anglo American [3]. Recentemente, o Vertimill também tem sido usado com sucesso nas etapas de moagem secundária e terciária [4]. A Figura 2 apresenta 0 circuito de remoagem do Sistema Minas-Rio que emprega Vertimill como equipamento de cominuição.

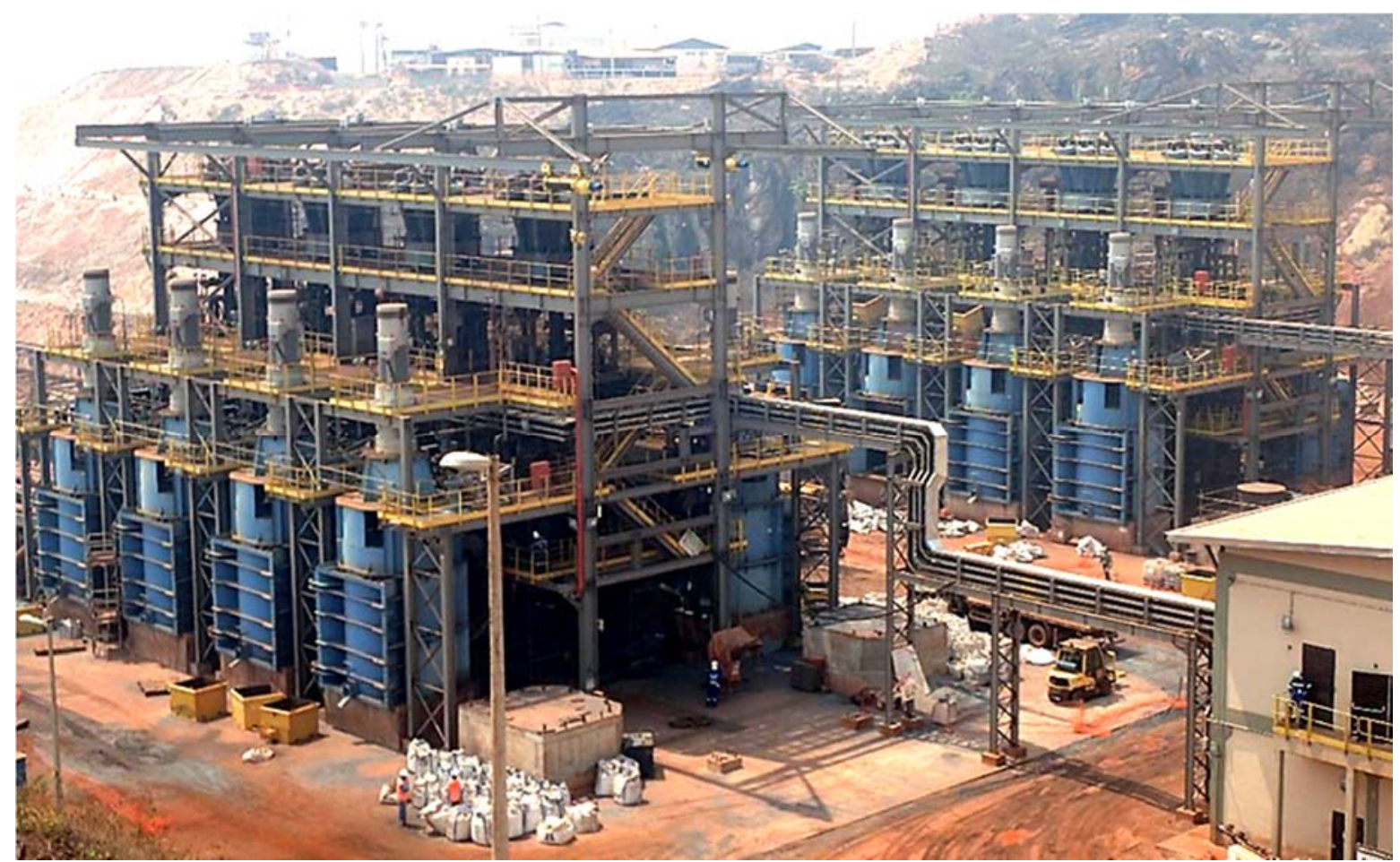

Figura 2. Circuito de remoagem com Vertimill do Minas-Rio.

A Figura 3 apresenta o layout do circuito de remoagem do Minas-Rio. O underflow de cada bateria de ciclonagem é dividido para alimentar dois moinhos Vertimill. Portanto, cada prédio tem quatro baterias de ciclones e oito moinhos Vertimill. Cada bateria de ciclone é composta por oito ciclones de $500 \mathrm{~mm}$ de diâmetro, totalizando 64 ciclones.

\subsection{Objetivo}

O objetivo deste trabalho é apresentar as primeiras impressões sobre o maior circuito de remoagem em operação do mundo utilizando Vertimill. O trabalho ainda apresenta as premissas adotadas no projeto e compara com os dados obtidos no circuito industrial até o momento. 


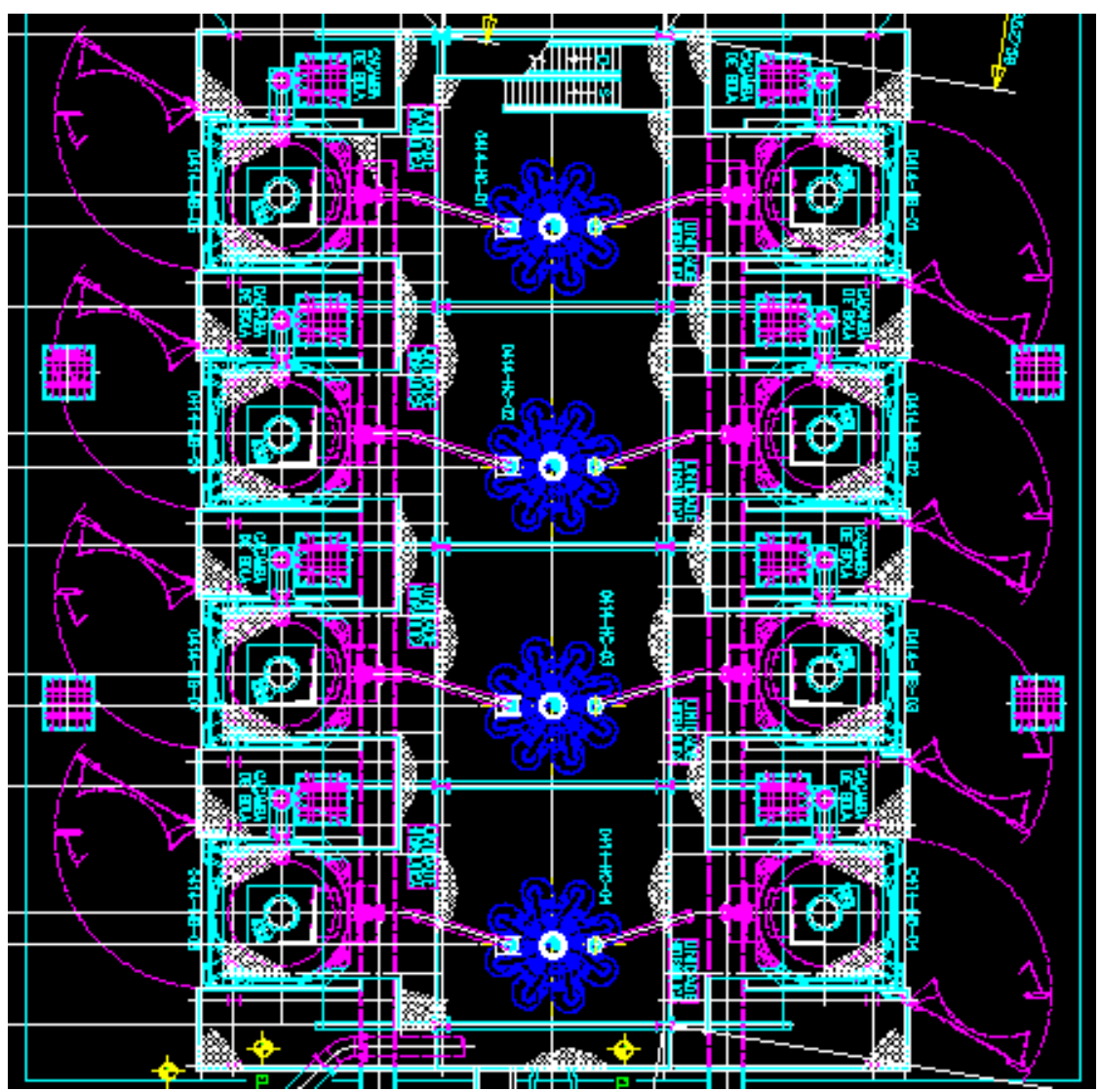

Figura 3. Layout (vista superior) do circuito de remoagem do Minas-Rio.

\section{MATERIAIS E MÉTODOS}

Os dados de taxa de alimentação do circuito de remoagem e de potência dos moinhos foram obtidos através do sistema de controle da planta. Amostradores automáticos se encontram instalados na alimentação e no produto do circuito de remoagem. A distribuição granulométrica do produto é medida e controlada para adequado bombeamento do Pellet Feed através do mineroduto.

Algumas amostragens do circuito de remoagem foram realizadas para verificação do desempenho dos moinhos e ciclones. Durante estas amostragens foram coletadas amostras da alimentação nova do circuito de remoagem utilizando-se o amostrador automático (concentrado proveniente da etapa de flotação). Foram coletadas alíquotas durante um período longo de forma a obter uma amostra representativa. Esta amostra foi utilizada para a execução de testes em escala de laboratório com o objetivo de estimar o consumo energético específico dos moinhos Vertimill.

A Figura 4 apresenta os fluxos amostrados e utilizados para fechamento do balanço de massas. 


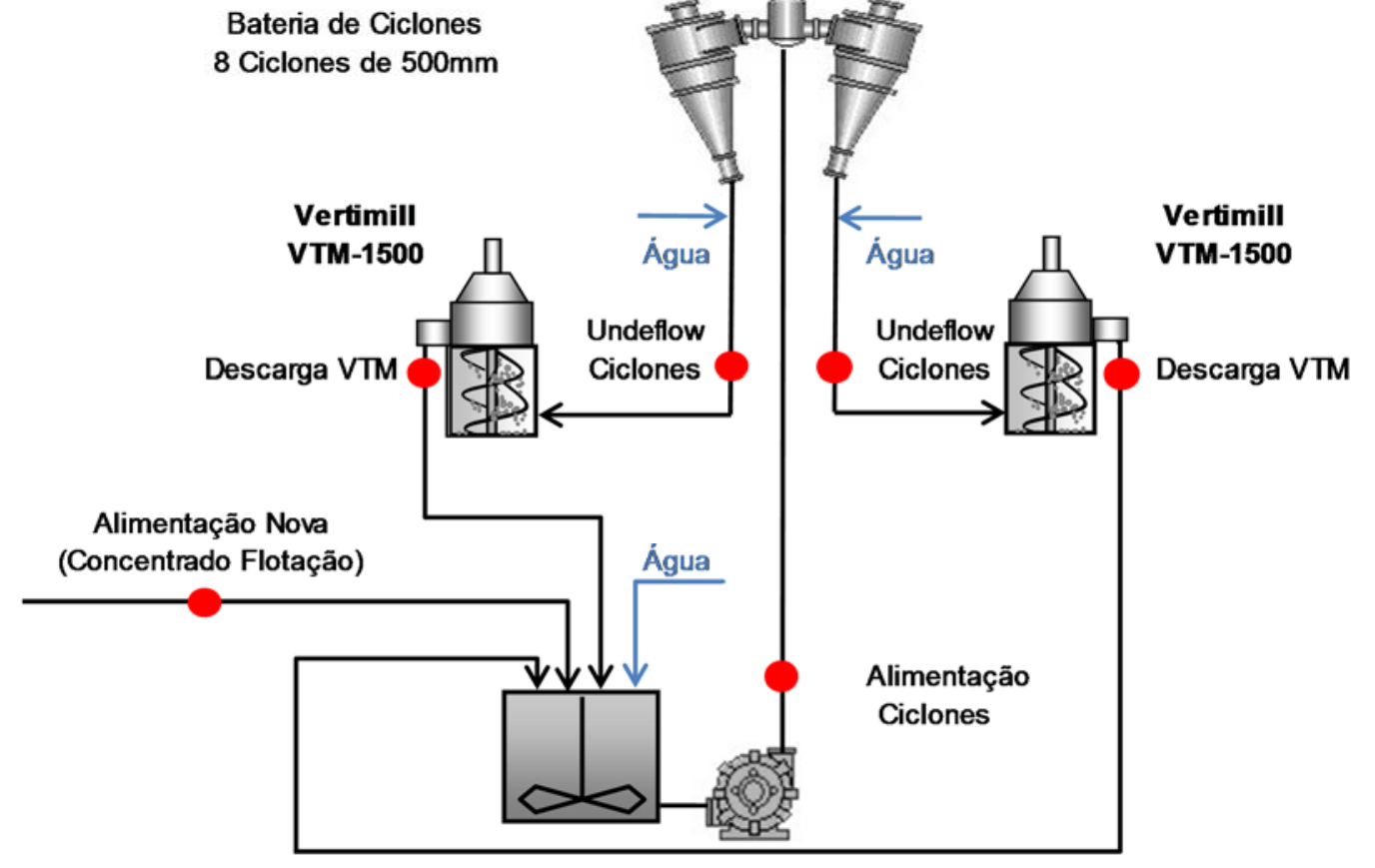

Figura 4. Pontos de amostragem.

Além da amostra da alimentação nova foram coletadas amostras da alimentação, underflow e overflow dos ciclones e da descarga do Vertimill. Para cada fluxo foi medida a distribuição granulométrica, inclusive da fração fina (Ciclosizer), e a concentração de sólidos em peso.

A Tabela 1 apresenta as condições operacionais adotadas no teste de moagem em escala de laboratório utilizando um moinho de bolas de batelada (Figura 5). No teste o moinho foi carregado com as mesmas bolas utilizadas nos moinhos Vertimill (bolas de alto cromo com $12,5 \mathrm{~mm}$ de diâmetro). Este teste foi executado com a alimentação nova do circuito de remoagem, tendo sido realizado nas mesmas condições que o teste realizado pela Metso para estimativa do consumo energético do Vertimill, conhecido com Jar Test. Normalmente são realizadas moagens em diferentes intervalos de tempo até se atingir o $P_{80}$ desejado. $O$ consumo energético obtido (kWh/t) é então multiplicado por um fator de 0,65 para se estimar o consumo energético do Vertimill.

Tabela 1 - Condições operacionais do teste de bancada com moinho de bolas

$\begin{array}{cc}\text { Diâmetro }(\mathrm{m}) & 0,208 \\ \text { Comprimento }(\mathrm{m}) & 0,208 \\ \text { Porosidade presumida da carga (\%) } & 40 \\ \text { Enchimento de Bolas }-\mathrm{J}(\%) & 42 \\ \text { Enchimento de Material - U (\%) } & 100 \\ \text { Fração da Velocidade Crítica (\%) } & 76 \\ \text { Concentração de Sólidos em Peso (\%) } & 70\end{array}$




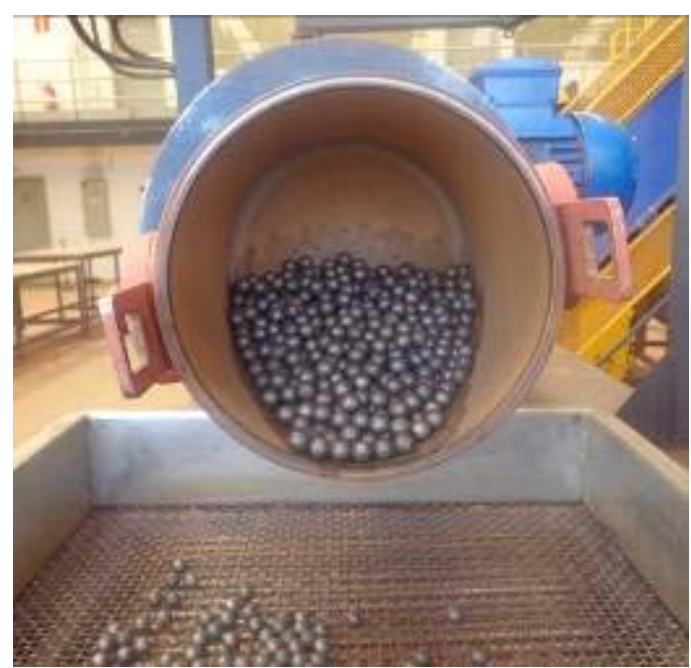

Figura 5. Teste de moagem em moinho de bolas de laboratório (Jar Test)

\section{RESULTADOS E DISCUSSÃO}

As análises granulométricas balanceadas obtidas a partir de uma das amostragens realizadas no circuito de remoagem são apresentadas na Figura 6.

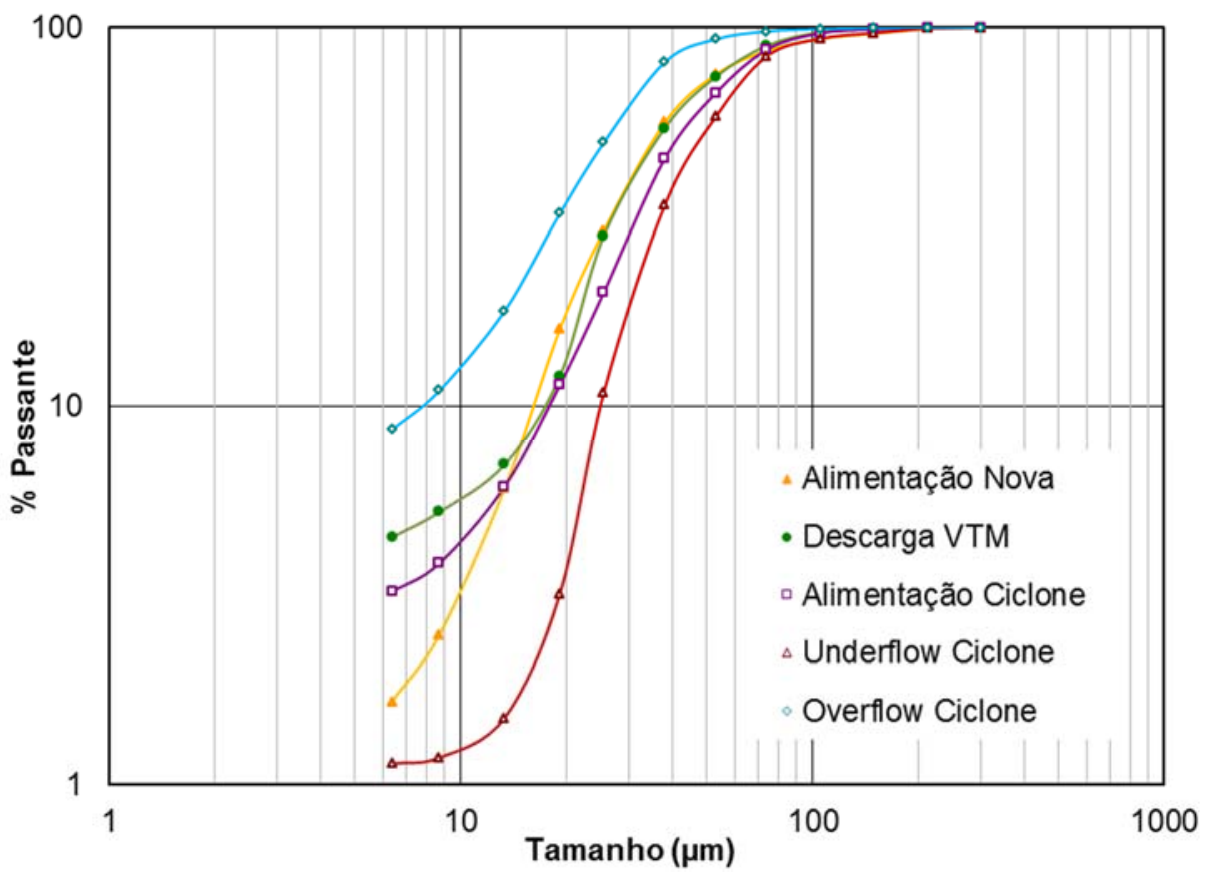

Figura 6. Análises granulométricas balanceadas.

A Figura 7 mostra o fluxo de polpa do undeflow em formato de leque sendo um indício de bom desempenho dos ciclones. 


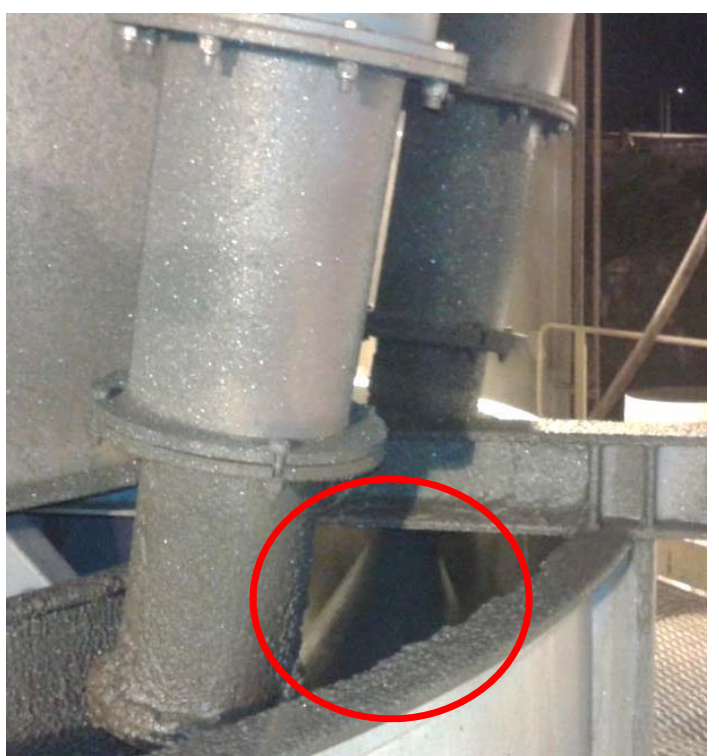

Figura 7. Amostragem do Undeflow dos ciclones

A Figura 8 apresenta as curvas granulométricas obtidas no teste de laboratório realizado com a amostra da alimentação nova do circuito de remoagem, assim como a Metso realizada o Jar Test. Foram realizadas três moagens nos tempos de 20, 40 e 60 minutos.

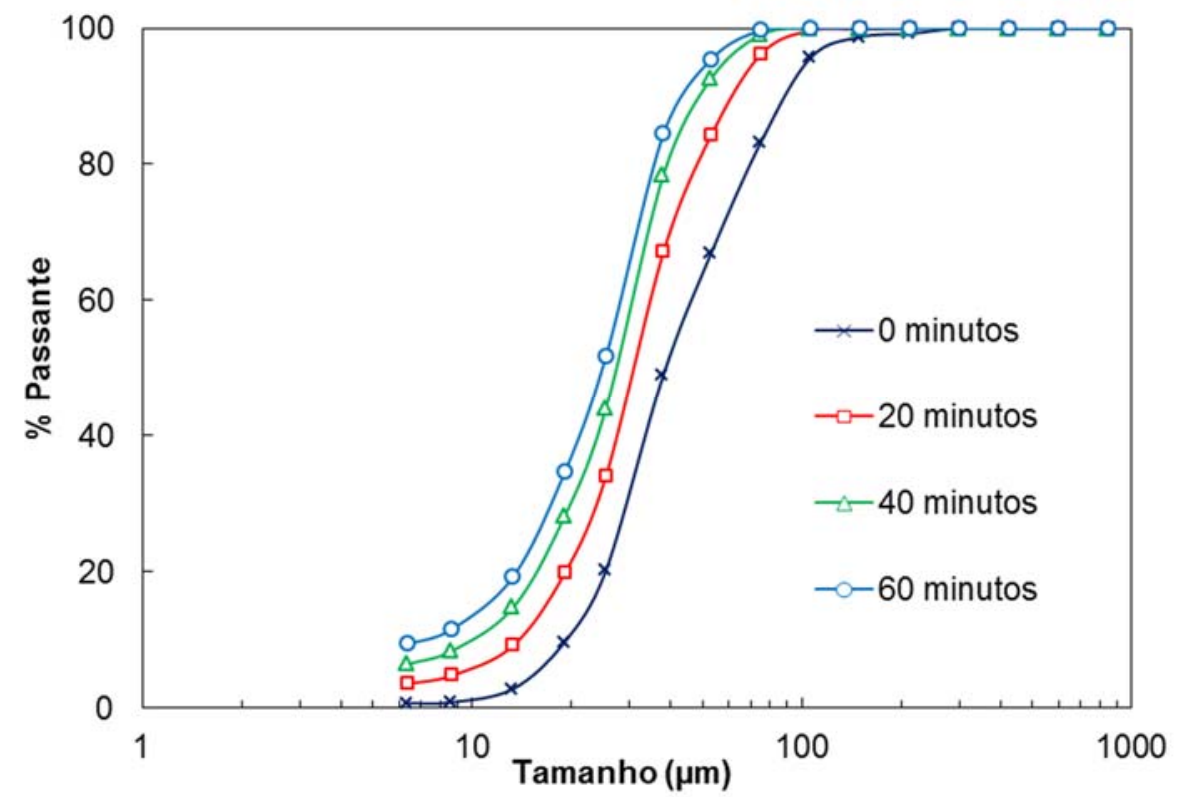

Figura 8. Curvas granulométricas obtidas no teste de moagem em laboratório

O último tempo de moagem (60 minutos) permitiu atingir o $P_{80}=36 \mu \mathrm{m}$ com um consumo energético de $8,9 \mathrm{kWh} / \mathrm{t}$. Adotando a metodologia da Metso para estimativa de consumo energético de moinho Vertimill, este valor de $8,9 \mathrm{kWh} / \mathrm{t}$ foi multiplicado por um fator de 0,65 para obter o consumo específico de 5,8 kWh/t para o Vertimill. Este valor está muito próximo ao valor adotado no projeto (16 VTM * $1119 \mathrm{~kW}=$ $17904 \mathrm{~kW} / 3040 \mathrm{t} / \mathrm{h}=5,9 \mathrm{kWh} / \mathrm{t}$ ), o que comprova a representatividade das amostras do circuito de remoagem empregadas no projeto. 
A Tabela 2 apresenta os dados obtidos nos testes em escala piloto utilizados para dimensionar o circuito de remoagem, as premissas de projeto e os dados obtidos na planta industrial. A especificação do produto $\left(P_{80}\right)$ está sendo alcançada.

Tabela 2 - Premissas de projeto e dados experimentais da planta industrial

\begin{tabular}{cccc}
\hline Dados & Piloto & Projeto & Atual \\
\hline$F_{80}(\mu \mathrm{m})$ & 66 & 70 & 62 \\
$P_{80}(\mu \mathrm{m})$ & 37 & 36 & 35 \\
Consumo energético específico $(\mathrm{kWh} / \mathrm{t})$ & 5,3 & 5,9 & 6,2 \\
\hline
\end{tabular}

Considerando que o Minas-Rio ainda se encontra na fase de ramp up é possível afirmar que existe espaço para otimização do circuito de remoagem.

\section{CONCLUSÃO}

O maior circuito de remoagem do mundo está operando bem até o momento e os dados obtidos por meio de amostragens e do controle de rotina do produto da remoagem mostram que o consumo energético dos moinhos Vertimill está próximo do consumo energético considerado na fase de projeto. Considerando que o MinasRio ainda se encontra na fase de ramp up é possível afirmar que existe espaço para otimização do circuito por meio do uso de diferentes corpos moedores e por ajustes nos hidrociclones. Os próximos passos são: continuidade da realização de amostragens no circuito para identificar possíveis melhorias e a avaliação do uso de diferentes corpos moedores com objetivo de manter/elevar a eficiência dos moinhos e reduzir os custos com este insumo.

\section{Agradecimentos}

Agradecimento especial a Anglo American por autorizar a publicação dos dados e promover assim a divulgação do uso de novas tecnologias aplicadas aos projetos minério de ferro.

\section{REFERÊNCIAS}

1. Mazzinghy, D.B. Metodologia para Escalonamento e Simulação de Moinho Vertical, Tese de Doutorado, PPGEM - Programa de Pós-Graduação em Engenharia Metalúrgica, Materiais e de Minas, UFMG, 2012.

2. Morrison, R.D., Cleary, P.W., Sinnott, M.D. Using DEM to compare the energy efficiency of pilot scale ball and tower mills, Minerals Engineering, 2009, 22: 665-672.

3. Bergerman, M.G. Dimensionamento e Simulação de Moinhos Verticais, Tese de Doutorado, Escola Politécnica da Universidade de São Paulo, 2013.

4. Palaniandy, S., Powell, M., Hilden, M., Allen, J., Kermanshahi, K., Oats, B., Lollback, M. VertiMill ${ }^{\circledR}$ - Preparing the feed within floatable regime at lower specific energy, Minerals Engineering, 2015, 73: 44-52. 